to infection. Similarly isolation at home and perhaps reduced visits from friends and relatives may have improved feeding success and explain the reduced admissions due to hypernatremic dehydration. Using TC services reduced the admission load on NICU, whilst also allowing mums and babies to stay together on the postnatal ward. In doing so, we will now review the readmission from home policy and capacity of TC to allow more babies to be kept together with their mums on re-admission, as we have demonstrated this can be done in a clinically safe manner. It is also a useful strategy for further waves of Covid-19, should there be more direct Covid-related admissions. With a reduction in community services we need to offer more support to parents with monitoring jaundice.

\section{A WEEKLY NEWSLETTER TO MAINTAIN TEAMWORK AND TEACHING}

Ruth Green, Meret Arsanious, Charles Stewart. UK

\subsection{6/bmjpo-2021-RCPCH.83}

Background With the emergence of the COVID-19 pandemic, the paediatric emergency department (PED) teaching was disrupted with the monthly half day education teaching mornings not taking place due to social distancing and reduced numbers of staff being allowed to be physically present on each shift. This lowered staff morale and reduced learning opportunities through the community of learners in the PED. This is particularly significant in a setting which manages a diverse range of pathologies, which has a high turnover of junior staff and where junior staff often face a steep learning curve at the start of their post, potentially posing increased clinical risk. A new teaching strategy was therefore implemented and delivered electronically in the form of a weekly 1-page newsletter.

Objectives To continue educating the staff within the PED

To maintain a positive morale within the PED team

Methods A weekly newsletter was sent out electronically to all staff within PED, it contained bitesize information on the PED topic of the week, signposting to further resources and an update on departmental news which included any new COVID-19 guidelines, local and regional training opportunities and celebrations of team-member's achievements and contributions to the department during that week. There was also a summary of learning outcomes from the departmental weekly simulation sessions and a section on learning from a real case that had recently been seen in the department. Additionally, there was a link to a weekly quiz which was predominantly based on the topic of the week, for staff members to assess their knowledge on the topic.

The topic of the week was based on common PED presentations. The quiz was used for participant's own self- assessment and this has been shown to be an effective learning strategy within education. Sharing real-life examples of cases and learning points from simulation allowed participants to connect with their learning by highlighting its relevance to the real-world. In addition, it provided the opportunity to safely reflect on their own practice (E.g what might they have done differently) and helped learners build across their 'zone of proximal development', thus giving the opportunity to develop cognitive skills and own world view.
Results Anonymised feedback was collected from a cross-section of department team members using an online survey 4 months after the intervention was started. The feedback received was that length and frequency of the newsletters was adequate, the quiz was positively received, and that team members felt that the newsletter brought a sense of comradery, despite social distancing.

Constructive feedback was given for future newsletters regarding its format to make it easier to read on mobile devices and suggestions of including notification of team-members' birthdays and junior staff to be involved in the newsletter's production, thus resulting in an even more inclusive construction of the newsletter.

Conclusions During the COVID-19 pandemic many areas of the department changed including teaching, however departmental learning and sense of team involvement was not diminished but rather thrived with the addition of the weekly paediatric emergency department newsletter.

\section{USING MULTIMODAL TEACHING METHODS TO IMPROVE CONFIDENCE AND ABILITY IN PERFORMING PAEDIATRIC EMERGENCY DEPARTMENT SKILLS}

Ruth Green, Meret Arsanious, Doug Macmaster, Charles Stewart. UK

\subsection{6/bmjpo-2021-RCPCH.84}

Background No published data exists regarding junior paediatric trainee's confidence in performing paediatric emergency department (PED) procedures but anecdotally this has been low in novices. In our hospital up to $60 \%$ of the $41,000 /$ year cases in PED are musculoskeletal ailments. There can be significant delays in treatment when junior team members are not able to independently perform PED procedures such as gluing and sizing crutches. Using multiple teaching methods appeals to different types of learners (e.g visual, auditory, kinesthetic learners) and using competition has been shown to encourage learning.

Objectives To improve junior paediatric staff member's confidence in performing specific PED skills before and after a multimodal teaching intervention is implemented.

Methods A curriculum was established and multimodal teaching of basic skills was started. This entailed procedural videos being created and circulated amongst new PED staff members with opportunities created to mentor them in practicing these skills. A star chart was used for participants to gauge their performance with their peers'. A competition was held at the end of the teaching intervention and trainees were asked to demonstrate these skills using objective criteria.

Results 26 participants took part. Participant confidence increased in $100 \%$ of cases in performing all the ED skills. Objective assessment of procedures post-intervention showed that all the participants were able to demonstrate performing the skills independently to a competent level. $100 \%$ of participants would recommend this intervention to others.

Conclusions The innovative multi-modal teaching intervention used was accepted by all learners, it was useful in improving junior paediatric staff member's confidence in performing certain PED procedures and to an objectively high level of competence. 